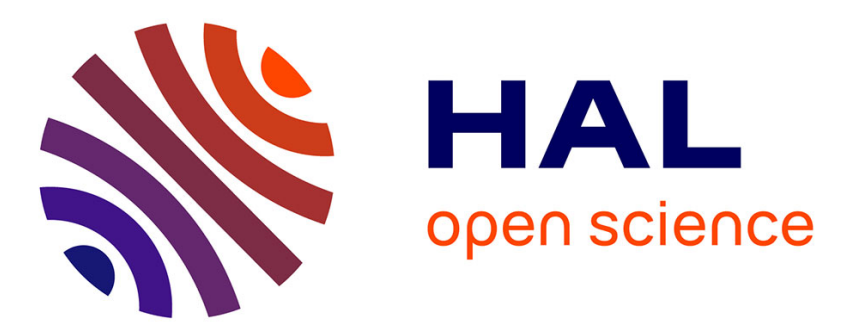

\title{
Simulation of shock-induced bubble collapse using a four-equation model
}

Eric Goncalves da Silva, Yannick Hoarau, Dia Zeidan

\section{To cite this version:}

Eric Goncalves da Silva, Yannick Hoarau, Dia Zeidan. Simulation of shock-induced bubble collapse using a four-equation model. Shock Waves, 2019, 29 (1), pp.221-234. 10.1007/s00193-018-0809-1 . hal-01715199

\author{
HAL Id: hal-01715199 \\ https://hal.science/hal-01715199
}

Submitted on 22 Feb 2018

HAL is a multi-disciplinary open access archive for the deposit and dissemination of scientific research documents, whether they are published or not. The documents may come from teaching and research institutions in France or abroad, or from public or private research centers.
L'archive ouverte pluridisciplinaire HAL, est destinée au dépôt et à la diffusion de documents scientifiques de niveau recherche, publiés ou non, émanant des établissements d'enseignement et de recherche français ou étrangers, des laboratoires publics ou privés. 


\title{
Simulation of shock-induced bubble collapse using a four-equation model
}

\author{
Eric Goncalves • Yannick Hoarau • Dia \\ Zeidan
}

Received: date / Accepted: date

\begin{abstract}
This paper presents a numerical study of the interaction between a planar incident shock wave with a cylindrical gas bubble. Simulations are performed using an inviscid compressible one-fluid solver based upon three conservation laws for the mixture variables, namely mass, momentum, and total energy along with a supplementary transport equation for the volume fraction of the gas phase. The study focuses on the maximum pressure generated by the bubble collapse. The influence of the strength of the incident shock is investigated. A law for the maximum pressure function of the Mach number of the incident shock is proposed.
\end{abstract}

Keywords Bubble collapse · Shock waves · Pressure peak · Jet formation

\section{Introduction}

The investigation of the pressure developed by a collapsing cavitation bubble is of primary interest for hydraulic turbomachinery, fuel injectors, naval propulsion systems and biomedical technology. One of the most critical consequences is the shock wave emitted by the collapse of bubbles and the structural damage resulting when this process takes place close to solid walls [29]. On the other hand, the destructive effects of cavitation can be exploited in medical application such as shock wave lithotripsy [18]. Since Lord Rayleigh, researchers have

E. Goncalves

ISAE-ENSMA, Institut Pprime, UPR 3346 CNRS, 86961 Futuroscope Chasseneuil, France E-mail: eric.goncalves@ensma.fr

Y. Hoarau

University of Strasbourg, ICUBE laboratory, UMR 7357 CNRS, 67000 Strasbourg, France E-mail: yannick.hoarau@unistra.fr

D. Zeidan

School of Basic Sciences and Humanities, German Jordanian University, Amman, Jordan E-mail: dia.zeidan@gju.edu.jo 
attempted to develop mathematical models in order to predict the bubble behaviour and response to hydrodynamic, thermal, or acoustic forcing.

To clarify the physical mechanism, numerous analytical, experimental [14, 7] and numerical studies of the collapse of a cavity in water under shock wave loading have been proposed. However, the high-speed dynamics, the small spatio-temporal scales as well as the complicated physics involved in these processes make any theoretical and experimental approach a challenge. Such shock-bubble interactions develop a liquid jet that penetrates through the bubble and emits a shock wave during the induced collapse. Both the impact of the jet and of the shock waves are possible damaging-generating mechanisms.

The numerical simulation of compressible two-phase fluid flows still exhibits severe difficulties: strong shock and rarefaction waves, topology changes, strong differences in material properties, non-equilibrium thermodynamics states, phase transition, etc. For the simulation of the interaction between a shock and bubbles, various approaches have been proposed in the literature: interfacetracking methods, direct simulation, averaged two-phase fluid flow models, or diffuse interface methods. All these approaches present advantages and drawbacks. With interface-tracking methods, the location of the interfaces, treated as sharp discontinuities, is explicitly represented during the time evolution. We can cite, for example, front-tracking methods [31], free-Lagrange methods [5] and level set/ghost fluid methods [23,16,27,22]. However, these methods encounter difficulties for large interface deformations and topology changes. To overcome this difficulty, techniques have been developed based on an Eulerian formulation whereby the location of the interface is captured implicitly. Diffuse interface methods are based on the solution of conservation laws (continuity, momentum, and energy equations), while relaxing the sharp character of material interfaces and thus allowing them to numerically diffuse over a small but finite region $[1,2,30,24,19,8,3]$. Within the averaged model family, there are different approaches according to the physical assumptions regarding thermodynamic equilibrium, and slip condition between phases. This has resulted in the development of various systems ranging from seven to three equations only. The two-fluid approach, e.g the model proposed by Baer and Nunziato [4], is the most complete and is also known to be a challenge for numerical simulation [33]. As an alternative way, a reduced five-equation model has been derived with the assumptions of velocity and pressure equilibrium $[20,26,9]$ and applied with success in various flows. By assuming the thermal equilibrium between phases, a four-equation model can be expressed. It is composed of three conservation laws for mixture quantities completed by an equation for a non-conservative quantity describing the flow topology, usually the void ratio $[21,11]$. With the assumption of complete thermodynamic equilibrium between phases (local temperature, pressure, and free Gibbs enthalpy equality between phases), the three-equation models or Homogeneous Equilibrium Models (HEM) are derived [12,34]. 
In the present study, the flow field resulting from the interaction between a planar incident shock wave and a circular gas bubble is numerically investigated. Simulations are performed using an inviscid compressible one-fluid code based on a four-equation system. It consists in solving three mixture conservation laws for the mass, momentum, and energy and a transport-equation for the gas volume fraction $[10,11]$. This four-equation model has been tested successfully on different cases including sheet cavitation, shock waves and underwater explosion. We describe the shock induced collapse, with particular attention on the maximum pressure reached during the process. The influence of the strength of the incident shock wave is investigated and attention is focused on the magnitude of the pressure peaks. The flow topology is detailed. The appearance of a recirculating area in front of the bubble is highlighted for a low intensity of the incident shock wave. An empirical law given the maximum pressure reached during the collapse as a function of the Mach number of the incident shock is proposed.

The paper is organized as follows. In section 2, we first review the theoretical formulation and models. The description of numerical methods is presented in section 3. Then, results are proposed in section 4 . Finally, conclusions and future investigations are discussed.

\section{Governing equations and models}

The numerical simulations are carried out using an in-house, two-phase code solving a one-fluid compressible inviscid system.

\subsection{The homogeneous approach}

The homogeneous mixture approach is used to model two-phase flows. The phases are assumed to be sufficiently well mixed and the sizes of the dispersed particle are sufficiently small, thereby eliminating any significant relative motion. The phases are strongly coupled and moving at the same velocity. In addition, the phases are assumed to be in thermal and mechanical equilibrium: they share the same temperature $T$ and the same pressure $P$. The evolution of the two-phase flow can be described by the conservation laws that employ the representative flow properties as unknowns just as in a single-phase problem. We introduce $\alpha_{k}$ the void fraction or the averaged fraction of presence of phase $k$. The density $\rho$, the center of mass velocity $u$, and the internal energy $e$ for the mixture are defined by [17]:

$$
\begin{aligned}
\rho & =\sum_{k} \alpha_{k} \rho_{k} \\
\rho u_{i} & =\sum_{k} \alpha_{k} \rho_{k} u_{i, k} \\
\rho e & =\sum_{k} \alpha_{k} \rho_{k} e_{k}
\end{aligned}
$$




\subsection{A four-equation model}

We consider a reduction of the five-equation Kapila model [20]. We assume that the liquid is at its saturation state. The model consists of three conservation laws for mixture quantities and an additional equation for the void ratio $\alpha$. Diffusion, surface tension, and phase change are not expected to affect the bubble dynamics over the major part of the collapse and are therefore ignored. We present below the inviscid two-dimensional equations, expressed in variables $U=(\rho, \rho \mathbf{V}, \rho E, \alpha)$ :

$$
\begin{aligned}
\frac{\partial \rho}{\partial t}+\operatorname{div}(\rho \mathbf{V}) & =0 \\
\frac{\partial(\rho \mathbf{V})}{\partial t}+\operatorname{div}\left(\rho \mathbf{V} \otimes \mathbf{V}+P I_{\mathrm{d}}\right) & =0 \\
\frac{\partial(\rho E)}{\partial t}+\operatorname{div}(\rho \mathbf{V} H) & =0 \\
\frac{\partial \alpha}{\partial t}+\mathbf{V} \cdot \operatorname{grad}(\alpha) & =\underbrace{\left(\frac{\rho_{\text {liq }} c_{\text {liq }}^{2}-\rho_{\text {vap }} c_{\text {vap }}^{2}}{\left.\frac{\rho_{\text {liq }} c_{\text {liq }}^{2}}{1-\alpha}+\frac{\rho_{\text {vap }} c_{\text {vap }}^{2}}{\alpha}\right)} \operatorname{div}(\mathbf{V})\right.}_{=K}
\end{aligned}
$$

where $\mathbf{V}=(u, v)$ is the center of mass velocity vector, $E=e+V^{2} / 2$ denotes the mixture total energy and $H=h+V^{2} / 2$ the mixture total enthalpy. Subscripts "liq" and "vap" for liquid and vapor, respectively. The term $K$ involves the speed of sound of pure phases $c_{k}$ and it reflects the effects of changes in volume of each phase.

\subsection{The mixture equation of state}

To close the system, an equation of state (EOS) is necessary to link the pressure and the temperature to the internal energy and the density. For the pure phases, we used the convex stiffened gas EOS (see [25]):

$$
P(\rho, e)=(\gamma-1) \rho(e-q)-\gamma P_{\infty} \quad \text { and } \quad T(\rho, h)=\frac{h-q}{C_{p}}
$$

where $\gamma=C_{p} / C_{v}$ is the heat capacity ratio, $C_{p}$ and $C_{v}$ are thermal capacities, $q$ the energy of formation of the fluid and $P_{\infty}$ is a constant reference pressure. The speed of sound $c$ is given by:

$$
c^{2}=\gamma \frac{P+P_{\infty}}{\rho}=(\gamma-1) C_{p} T
$$

On the basis of the stiffened gas EOS for each pure phase, an expression for the pressure and the temperature can be deduced from the thermal and 
mechanical equilibrium assumption. It is worth noting that these expressions are available in all possible fluid states along with the function of the void ratio $\alpha$ and the mass fraction of gas $Y=\alpha \rho_{\mathrm{vap}} / \rho$ :

$$
\begin{aligned}
P(\rho, e, \alpha, Y) & =(\gamma(\alpha)-1) \rho(e-q(Y))-\gamma(\alpha) P_{\infty}(\alpha) \\
\frac{1}{\gamma(\alpha)-1} & =\frac{\alpha}{\gamma_{\text {vap }}-1}+\frac{1-\alpha}{\gamma_{\text {liq }}-1} \quad ; \quad q(Y)=Y q_{\text {vap }}+(1-Y) q_{\text {liq }}( \\
P_{\infty}(\alpha) & =\frac{\gamma(\alpha)-1}{\gamma(\alpha)}\left[\alpha \frac{\gamma_{\text {vap }}}{\gamma_{\text {vap }}-1} P_{\infty}^{\text {vap }}+(1-\alpha) \frac{\gamma_{\text {liq }}}{\gamma_{\text {liq }}-1} P_{\infty}^{\text {liq }}\right] \\
T(\rho, h, Y) & =\frac{h-q(Y)}{C_{p}(Y)} \text { with } C_{p}(Y)=Y C_{p_{\text {vap }}}+(1-Y) C_{p_{\text {liq }}}
\end{aligned}
$$

where more about the above parameters will be given in later sections.

Further, the four equations form a system of conservation laws having a hyperbolic nature [11]. The eigenvalues of the system are:

$\lambda_{1}=u-c_{\text {wallis }}, \lambda_{2,3,4}=u$ and $\lambda_{5}=u+c_{\text {wallis }}$ where $c_{\text {wallis }}$ is the propagation of acoustic waves without mass and heat transfer. This speed of sound is expressed as a weighted harmonic mean of speeds of sound of each phase:

$$
\frac{1}{\rho c_{\mathrm{wallis}}^{2}}=\frac{\alpha}{\rho_{\mathrm{vap}} c_{\mathrm{vap}}^{2}}+\frac{1-\alpha}{\rho_{\mathrm{liq}} c_{\mathrm{liq}}^{2}} .
$$

\section{Numerics}

The numerical simulations are carried out using an explicit solver for structured meshes. This solver is based on a cell-centered finite-volume discretisation $[13]$.

\subsection{Space discretisation}

In two-dimensional space, the four-equation model can be represented in a matrix form as:

with

$$
\frac{\partial U}{\partial t}+\operatorname{div}[G(U)]+B(U) \operatorname{div} \mathbf{V}=0
$$

$U=\left(\begin{array}{c}\rho \\ \rho \mathbf{V} \\ \rho E \\ \alpha\end{array}\right) \quad ; \quad G(U)=\left(\begin{array}{c}\rho \mathbf{V} \\ \rho \mathbf{V} \otimes \mathbf{V}+P I_{d} \\ \rho H \mathbf{V} \\ \alpha \mathbf{V}\end{array}\right) ; \quad B(U)=\left(\begin{array}{c}0 \\ \mathbf{0} \\ 0 \\ -(K+\alpha)\end{array}\right)$

We focus herein on finite volume schemes. Regular meshes are considered. Integrating the system on the cell $\mathcal{C}_{i}$ gives

$$
\Omega_{i} \frac{\partial U_{i}}{\partial t}+\sum_{l \in \partial \mathcal{C}_{i}} \int_{l} G(U) \cdot \mathbf{n}_{i, l} d l+\int_{\mathcal{C}_{i}} B(U) \operatorname{div} \mathbf{V} d S=0
$$


where $\partial \mathcal{C}_{i}$ is the boundary of the cell, $\Omega_{i}$ the surface of the cell and $\mathbf{n}_{i, l}$ is the outward normal (with respect to cell $\mathcal{C}_{i}$ ) of cell interface $l$.

The numerical flux through the cell interface is computed with a HLLC scheme $[32,6]$. The method considers two averaged intermediate states $U_{L}^{*}$ et $U_{R}^{*}$ separated by the contact wave of speed $S_{M}$. The numerical flux $\Phi_{i, l}$ at cell interface $l$ can be expressed as:

$$
\Phi_{i, l}\left(U_{L}, U_{R}\right)=\left\{\begin{array}{lll}
G\left(U_{L}\right) \mathbf{n}_{i, l} & \text { if } & S_{L}>0 \\
G\left(U_{L}^{*}\right) \mathbf{n}_{i, l} & \text { if } & S_{L} \leq 0<S_{M} \\
G\left(U_{R}^{*}\right) \mathbf{n}_{i, l} & \text { if } & S_{M} \leq 0 \leq S_{R} \\
G\left(U_{R}\right) \mathbf{n}_{i, l} & \text { if } & S_{R}<0
\end{array}\right.
$$

where $S_{L}$ and $S_{R}$ are referred to the speeds of the smallest and largest waves at the cell interface.

We introduce the normal velocity component $V_{n}=$ V.n. The left $(K=L)$ and right $(K=R)$ states of the variables $U_{K}^{*}$, and corresponding fluxes $G\left(U_{K}^{*}\right)$, are defined by:

$$
\begin{gathered}
U_{K}^{*}=\left(\begin{array}{c}
\rho_{K}^{*} \\
(\rho u)_{K}^{*} \\
(\rho v)_{K}^{*} \\
(\rho E)_{K}^{*} \\
\alpha_{K}^{*}
\end{array}\right)=\frac{1}{S_{K}-S_{M}}\left(\begin{array}{c}
\rho_{K}\left(S_{K}-V_{n_{K}}\right) \\
(\rho u)_{K}\left(S_{K}-V_{n_{K}}\right)+\left(P^{*}-P_{K}\right) n_{i, l} \\
(\rho u)_{K}\left(S_{K}-V_{n_{K}}\right)+\left(P^{*}-P_{K}\right) n_{i, l} \\
(\rho E)_{K}\left(S_{K}-V_{n_{K}}\right)+P^{*} S_{M}-P_{K} V_{n_{K}} \\
\alpha_{K}\left(S_{K}-V_{n_{K}}\right)
\end{array}\right) \\
G\left(U_{K}^{*}\right) \mathbf{n}_{i, l}=\left(\begin{array}{c}
(\rho u)_{K}^{*} S_{M}^{*} S_{M}+P^{*} n_{i, l} \\
(\rho v)_{K}^{*} S_{M}+P^{*} n_{i, l} \\
(\rho E)_{K}^{*} S_{M}+P^{*} S_{M} \\
\alpha_{K}^{*} S_{M}
\end{array}\right)
\end{gathered}
$$

where the pressure $P^{*}$ is given by:

$$
P^{*}=P_{L}+\rho_{L}\left(V_{n_{L}}-S_{L}\right)\left(V_{n_{L}}-S_{M}\right)=P_{R}+\rho_{R}\left(V_{n_{R}}-S_{R}\right)\left(V_{n_{R}}-S_{M}\right)
$$

And the contact-wave speed $S_{M}$ is defined by:

$$
S_{M}=\frac{P_{R}-P_{L}+\rho_{L} V_{n_{L}}\left(S_{L}-V_{n_{L}}\right)-\rho_{R} V_{n_{R}}\left(S_{R}-V_{n_{R}}\right)}{\rho_{L}\left(S_{L}-V_{n_{L}}\right)-\rho_{R}\left(S_{R}-V_{n_{R}}\right)}
$$

The HLLC solver requires the estimates of wave speeds $S_{L}$ and $S_{R}$ in the Riemann problem. A direct and simple wave speed estimation is used:

$$
S_{L}=\operatorname{Min}\left(V_{n_{L}}-c_{L}, V_{n_{R}}-c_{R}\right) \quad ; \quad S_{R}=\operatorname{Max}\left(V_{n_{L}}+c_{L}, V_{n_{R}}+c_{R}\right)
$$

The non-conservative term is discretized following the idea of Daude et al. [9]. The integral term is approximated with the following relation:

$$
\int_{\mathcal{C}_{i}} B(U) \operatorname{div} \mathbf{V} d S=\tilde{B}_{i} \sum_{l \in \partial \mathcal{C}_{i}} \int_{\partial \mathcal{C}_{i}} \mathbf{V} \cdot \mathbf{n}_{i, l} d l
$$


where $\tilde{B}_{i}$ is some average of $B$ on cell $\mathcal{C}_{i}$. In this work, we have used $\tilde{B}_{i}=B\left(U_{i}\right)$.

The cell interface value $u_{i, l}$ is expressed as:

$$
u_{i, l}\left(U_{L}, U_{R}\right)=\left\{\begin{array}{cll}
\mathbf{V}_{L} \cdot \mathbf{n}_{i, l} & \text { if } & S_{L}>0 \\
\frac{S_{L}-V_{n}}{S_{L}-S_{M}} S_{M} & \text { if } & S_{L} \leq 0<S_{M} \\
\frac{S_{R}-V_{n}}{S_{R}-S_{M}} S_{M} & \text { if } & S_{M} \leq 0 \leq S_{R} \\
\mathbf{V}_{R} \cdot \mathbf{n}_{i, l} & \text { if } & S_{R}<0
\end{array}\right.
$$

The second-order accuracy in space is obtained using the MUSCL approach. The minmod slope limiter is used with the MUSCL extrapolation. The temporal integration is performed using a first-order forward scheme.

\subsection{Inlet and outlet boundary conditions}

The numerical treatment of the boundary conditions is based on the use of the characteristic relations of the Euler equations. The number of variables to impose at boundaries is given by the number of positive characteristics directed into the domain of interest. The characteristic relations obtained for the four-equation system, in two-dimensional flows, are:

$$
\begin{aligned}
-c^{2}\left(\rho^{c}-\rho^{s}\right)+\left(P^{c}-P^{s}\right) & =0 \\
V_{t}^{c}-V_{t}^{s} & =0 \\
\rho\left(\alpha^{c}-\alpha^{s}\right)-K\left(\rho^{c}-\rho^{s}\right) & =0 \\
\left(P^{c}-P^{s}\right)+\rho c\left(V_{n}^{c}-V_{n}^{s}\right) & =0 \\
\left(P^{c}-P^{s}\right)-\rho c\left(V_{n}^{c}-V_{n}^{s}\right) & =0
\end{aligned}
$$

The variables with superscript $c$ denote the variables to be computed at the boundary. Variables with superscript $s$ denote the variables obtained by the current numerical scheme. $V_{t}$ and $V_{n}$ are the tangential and the normal component of the velocity, respectively.

At inflow, we impose the initial values of the void ratio, densities of pure phases, and the velocity. The pressure is evaluated with the relation (27) and all variables can be evaluated at the boundary.

At the outflow, the static pressure is imposed. The variables are computed with four characteristic relations (23)-(26).

\section{Simulation results}

4.1 The reference case: $\Delta P_{\mathrm{sh}}=1.910^{9} \mathrm{~Pa}, M_{\mathrm{sh}}=1.72$

The reference test case is similar to the one presented in [5,27]. A cylindrical air bubble, for which the initial diameter $D_{0}=6 \mathrm{~mm}$, is immersed in a water pool, under the following initial conditions: $\mathbf{V}=(0,0) \mathrm{m} / \mathrm{s}, P=10^{5} \mathrm{~Pa}$, 
$\rho_{\text {air }}=1 \mathrm{~kg} / \mathrm{m}^{3}$, and $\rho_{\text {water }}=1000 \mathrm{~kg} / \mathrm{m}^{3}$. Due to the symmetry of the problem the calculations are performed in a half-domain above the axis. The center of the bubble is located at $(9,0) \mathrm{mm}$ in the computational domain of size $24 \times 12 \mathrm{~mm}$. The bubble is collapsed by a normal shock wave moving at $M_{\mathrm{sh}}=1.72$, initially located at abscissa $x_{\mathrm{sh}}=4 \mathrm{~mm}$. A schematic diagram of the test case is given in Figure 1. Parameters of the EOSs and post-shock conditions are:

$$
\begin{gathered}
\left(\begin{array}{c}
\gamma \\
P_{\infty} \\
\rho
\end{array}\right)_{\text {Liquid }}=\left(\begin{array}{c}
4.4 \\
6 \times 10^{8} \mathrm{~Pa} \\
1000 \mathrm{~kg} / \mathrm{m}^{3}
\end{array}\right) \quad ; \quad\left(\begin{array}{c}
\gamma \\
P_{\infty} \\
\rho
\end{array}\right)_{\mathrm{Gas}}=\left(\begin{array}{c}
1.4 \\
0 \mathrm{~Pa} \\
1 \mathrm{~kg} / \mathrm{m}^{3}
\end{array}\right) \\
\left(\begin{array}{c}
\mathrm{P} \\
\rho \\
\mathrm{u}
\end{array}\right)_{\text {post-shock }}=\left(\begin{array}{c}
1.910^{9} \mathrm{~Pa} \\
1323.65 \mathrm{~kg} / \mathrm{m}^{3} \\
681.58 \mathrm{~m} / \mathrm{s}
\end{array}\right)
\end{gathered}
$$

The boundary conditions are the following: The top and bottom boundaries are assumed to be a wall and a symmetry axis, respectively. The left and right sides are assumed to be non-reflecting. Simulations are performed using a uniform mesh and a time step $\Delta t=10^{-9} \mathrm{~s}$.

A preliminary study on the effect of spatial resolution was conducted to ensure that grid independence was achieved. Resolutions tested varied from 400 to 2800 nodes in the longitudinal direction corresponding to 100 to 700 points per bubble diameter (ppbd). To evaluate the grid convergence, two quantities are presented in Figure 2: the intensity of the pressure peaks and the time corresponding to the maximum pressure peak. The plotted values have been normalized by the value obtained using the finest grid. For the time evolution, it is shown that a resolution of 200 ppbd is sufficient (around 1\% of difference with the finest grid). Similarly, the first pressure peak converges rapidly with the grid resolution. More variation is illustrated for the second pressure peak. Based on this study, a resolution of $300 \mathrm{ppbd}$ was viewed sufficient to capture the main features of the bubble collapse (around $5 \%$ of difference for the second pressure peak). The influence of the time step was done using the chosen grid composed by $1200 \times 600$ nodes. Based on the initial value of $1 \mathrm{~ns}$, simulations were performed using a time step varying from $0.125 \mathrm{~ns}$ to $2 \mathrm{~ns}$. The maximum pressure intensity normalized by the value obtained with the smallest time step is plotted in Figure 3. Unlike the grid refinement, the pressure peak decreases when the time step is reduced. In view of this study, the value of $1 \mathrm{~ns}$ was conserved for all simulations.

Firstly, a description of the main phenomena involved in this shock-bubble interaction is proposed. The time evolution of the density gradient modulus (Schlieren-type representation) andthe pressure field are plotted in Figure 4 from time $t=2 \mu \mathrm{s}$ to $t=5.2 \mu \mathrm{s}$. After the water shock wave has collided with the bubble, a strong rarefaction wave is reflected backwards from the 
interface, and a weak shock wave is transmitted inside of the bubble (time $t=2 \mu \mathrm{s})$. Due to the pressure difference between both sides, the bubble is asymmetrically contracted and spreads laterally in the process. As time moves on, the bubble becomes kidney shaped and spreads laterally in the process. This change in shape is driven by vorticity generated at the edge of the bubble due to the passage of the wave, which induces a jet of water along the axis of flow symmetry. When this water jet impacts the stationary water at the front of the bubble (at time $t=3.6 \mu \mathrm{s}$ ), an intense blast wave (referred to as waterhammer shock [15]) is formed generating a high-pressure zone. The blast front, which expands continuously, is highly asymmetric due to the high-speed water jet (see at time $t=4.2 \mu \mathrm{s}$ ). The rightward blast wave increases as a spherical wave. Both shocks lose strength as they advance, the rightward wave more so than its leftward twin. The interaction of the blast wave with the bubble fragments leads to high pressure levels (at time $t=4.8 \mu \mathrm{s}$ ), which is the most intense reached during the collapse. Finally, at time $t=5.2 \mu \mathrm{s}$, the blast wave continues its expansion and the cavity its shrinkage. The low-pressure areas inside the core of the vortices are well illustrated. These results show a good agreement with previous numerical results $[5,27,28]$.

The time evolution of the axial velocity is plotted in Figure 5. The reflected rarefaction wave, resulting from the impact of the incident shock with the upstream bubble interface, relaxes the pressure, which accelerates the flow and forms a high-speed water jet (time $t=2 \mu \mathrm{s}$ and after). The velocity magnitude is higher than $2000 \mathrm{~m} / \mathrm{s}$. At time $t=3.6 \mu \mathrm{s}$, the water jet strikes the downstream bubble interface leading to the blast wave generation. After time $t=3.8 \mu \mathrm{s}$, the bubble is cut in half and forms a pair of distinct vortical structures. The developing leftward wave advances relatively slowly due to the high water velocity in the jet fluid. At time $t=4.6 \mu \mathrm{s}$, the front of the leftward wave can be observed (abscissa $x \simeq 0.012 \mathrm{~m}$ ).

The pressure evolution on the axis and the dimensionless maximum pressure $P_{\max } / \Delta P_{\text {sh }}$ in the domain, plotted in Figure 6 , illustrate the high pressure reached during the cavity collapse. We can observe the first peak at time $t=3.8 \mu \mathrm{s}$ after the water jet impacts the bubble front leading to the blast wave generation and the second peak (more intense, around 78000 bar) at time $t=4.8 \mu$ s when the leftward blast wave collides with the bubble fragments. For the first pressure peak, oscillations of pressure are observed due to the fact that the maximum pressure value oscillates on different cells near the axis. In comparison with previous simulations, we obtain an intermediate value between results of Ball et al. - 4.7 GPa [5] -dash and Nourgaliev et al. -dash 10.1 GPa [27].

The evolution of both the axial velocity (left) and the temperature on the symmetry axis (right) during the bubble collapse is shown in Figure 7. The acceleration of the flow leading to the high-speed jet is clearly illustrated. At time $t=3.6 \mu \mathrm{s}$, when the liquid jet impacts the downstream bubble interface, 
the axial velocity reached its maximum value around $2820 \mathrm{~m} / \mathrm{s}$. This value is very close to the one obtained by Nourgaliev et al. - $2850 \mathrm{~m} / \mathrm{s}$ [27]. After this time, the jet velocity slightly decreases and remains at a high level around $2500 \mathrm{~m} / \mathrm{s}$.

The temperature evolution along the axis clearly highlights two intense peaks at times 2 and $3 \mu \mathrm{s}$, respectively. They correspond to the temperature augmentation across the transmitted shock wave inside the bubble. The more intense peak (higher than $10000 \mathrm{~K}$ ) is observed at time $t=3.4 \mu$ s just before the high-speed jet impinges upon the downstream side of the bubble. Such extreme values leading to the formation of a hot plasma have been discussed in studies on the sonoluminescence phenomenon. The maximum temperature reached in the simulations of Ball et al. and Nourgaliev et al. is about $12000 \mathrm{~K}$ and $25000 \mathrm{~K}$, respectively.

4.2 Second case: $\Delta P_{\mathrm{sh}}=1.9 \cdot 10^{8} \mathrm{~Pa}, M_{\mathrm{sh}}=1.1$

We consider a similar case involving a weaker shock wave. The initial diameter is $D_{0}=5 \mathrm{~mm}$. The bubble is collapsed by a normal shock wave moving at $M_{\mathrm{sh}}=1.1$, initially located at abscissa $x_{\mathrm{sh}}=4 \mathrm{~mm}$. Post-shock conditions are:

$$
\left(\begin{array}{l}
\mathrm{P} \\
\rho \\
\mathrm{u}
\end{array}\right)_{\text {post-shock }}=\left(\begin{array}{c}
1.910^{8} \mathrm{~Pa} \\
1064.08 \mathrm{~kg} / \mathrm{m}^{3} \\
106.95 \mathrm{~m} / \mathrm{s}
\end{array}\right)
$$

For this case, the size of the computational domain is $20 \times 10 \mathrm{~mm}$. The resolution involves 300 nodes per bubble diameter and the time step is $\Delta t=10^{-9} \mathrm{~s}$.

Firstly, a description of the main phenomena involved in this interaction is proposed. The evolution of the density gradient modulus, the pressure field, and the axial velocity at different times (from $t=3 \mu \mathrm{s}$ to $t=9.4 \mu \mathrm{s}$ ) are plotted in Figure 8. As described previously, after the incident shock wave has collided with the bubble, a strong rarefaction wave is generated and a weak shock wave is transmitted inside the bubble. For this case, the incident shock wave is largely faster than the transmitted wave in the gas. The incident shock has reached the midpoint of the bubble at time $t=3 \mu$ s and the downstream part of the bubble at time $t=5 \mu \mathrm{s}$. At time $t=7 \mu \mathrm{s}$, the transmitted shock reached the midpoint of the bubble whereas the incident shock has propagated beyond the bubble and reflected off the axis. As the liquid jet forms, it impacts the downstream bubble interface producing a strong water-hammer shock wave (at time $t=9.4 \mu \mathrm{s}$ and after) leading to a high pressure area. Moreover, the pressure contours show that inside the reduced pressure area (due to the rarefaction wave), an oblique pressure gradient develops. As a consequence, the flow entering the rarefaction front converges toward the bubble interface. This phenomenon is clearly observed in the plot of the streamlines. At time $t=5 \mu \mathrm{s}$, a reverse flow is highlighted, which passes though the inter- 
face and penetrates inside the bubble. A saddle point can be seen along the axis downstream of the bubble at location $x=0.0113 \mathrm{~m}$. During the same time, the axial velocity increases forming the high-speed jet. At time $t=5 \mu \mathrm{s}$, the streamlines indicate the reversal of the flow, which enters inside the bubble and converges just behind the transmitted shock wave. The saddle point on the axis moves downstream at abscissa $x=0.013 \mathrm{~m}$. At time $t=9.4 \mu \mathrm{s}$, the reverse flow converges toward the rightward blast wave front. A focus point exists just downstream of the blast wave front (not clearly visible on figure) at position $(x, y)=(0.0103,-0.00075)$.

The same quantities are plotted in Figure 9 from time $t=9.8 \mu \mathrm{s}$ to $t=11.4 \mu \mathrm{s}$. Features described previously for the case $M_{\mathrm{sh}}=1.72$ are present. One main difference is that the rightward blast wave front propagates in the pressure field behind the incident shock, in a high-pressure environment. The rightwardtraveling shock increases as a spherical wave and propagates faster than its leftward twin. The interaction of the leftward-traveling shock with the bubble fragments lead to high pressure levels (at time $t=10.4 \mu \mathrm{s}$ ). At this stage the bubble is transformed into two elongated parts with counter-rotating vortices (clearly illustrated by the streamlines plot) building a thin channel with the high velocity jet flowing in between. Due to the extension of the vortical structures and their contouring close to the axis, this thin channel narrows and, at time $t=10.8 \mu \mathrm{s}$, the high-speed jet is cut into two parts. The deceleration of the axial flow between the two jets is very abrupt (by a factor ten, from $350 \mathrm{~m} / \mathrm{s}$ to $35 \mathrm{~m} / \mathrm{s}$ ) and a small low velocity area can be observed on the axis (abscissa $x=0.0092 \mathrm{~m}$ ). Simultaneously, the leftward blast wave front focuses with the spherical wave and propagates against the first jet flow. A strong peak pressure is generated behind the leftward shock due to the low speed area. This high pressure level is the most intense reached during the collapse. The intensity of the first jet progressively decreases as the leftward shock propagates. At the same time, the low velocity area extends and negative axial values are reached. At time $t=11.4 \mu \mathrm{s}$, the first jet vanishes as the blast wave propagates. A recirculation area is clearly visible just behind the leftward wave front. On the other side, the rightward wave propagates radially and streamlines converge along the wave front. The saddle point located downstream of the wave at time $t=10.8 \mu$ s disappears when the shock wave passes.

The evolution of both the pressure on the symmetry axis (left) and the dimensionless maximum pressure (right) during the collapse is plotted in Figure 10. At time $t=9.4 \mu \mathrm{s}$, the water jet strikes the downstream bubble interface generating the blast wave, leading to the pressure increase. A first pressure peak is observed at time $t=10 \mu \mathrm{s}$ on the axis (around 9000 bar) due to the propagation of the leftward wave. At time $t=10.4 \mu \mathrm{s}$ a second pressure peak (around 14000 bar) is generated when the leftward wave interacts with the bubble pieces. On the axis, the pressure level has the same magnitude of the first peak. At time $t=10.8 \mu \mathrm{s}$, a third peak is illustrated on the axis. It is the most important pressure peak during the collapse (around 16000 bar). As discussed previously, it is due to the existence of a low velocity area along the 
axis just behind the leftward wave front.

Evolutions of the axial velocity and the temperature along the axis (plotted with a logarithmic scale) are drawn in Figure 11. The acceleration of the flow leading to the jet formation is clearly observed. The maximum velocity (around $1000 \mathrm{~m} / \mathrm{s}$ ) is reached at time $t=9.4 \mu \mathrm{s}$ when the jet impacts the downstream part of the bubble and remains at this level up to time $t=10 \mu \mathrm{s}$. Downstream of the bubble, the backflow is illustrated with negative values of the velocity. At time $t=10.8 \mu \mathrm{s}$, the front of the leftward wave is visible (abscissa $x=0.0095 \mathrm{~m}$ ) and also two peaks of velocity corresponding to the jet cutting. At time $t=11.4 \mu \mathrm{s}$, a small area of negative velocity is highlighted just behind the leftward wave front. Concerning the temperature evolution along the axis, a first peak (around $2000 \mathrm{~K}$ ) is illustrated at time $t=8 \mu$ s during the large reduction of the bubble volume. The most intense peak (around $8000 \mathrm{~K}$ ) is observed at time $t=9.4 \mu \mathrm{s}$ when the liquid jet impacts the downstream interface of the bubble.

\subsection{Influence of the shock intensity on the pressure peak}

Different simulations are performed by varying the intensity of the incident shock from $M_{\mathrm{sh}}=1.1$ to 1.9 .

The third peak and the presence of a recirculating area are observed only at low Mach number of the incident shock: $M_{\mathrm{sh}}=1.1,1.15$ and 1.2. We focus on the value of the maximum pressure peak reached during the collapse. For all cases, the dimensionless maximum pressure $P_{\max }^{*}=P_{\max } / \Delta P_{\mathrm{sh}}$ is reported in Table 4.3. As mentioned previously, this maximum value is stronger at lower shock intensities. We introduce the dimensionless maximum axial velocity $U_{\max }^{*}=U_{\max } / U_{\mathrm{sh}}$, given in Table 4.3 . We observed similarly that this quantity decreases as the shock intensity increases.

\begin{tabular}{lllll}
\hline$M_{\mathrm{sh}}$ & $\Delta P_{\mathrm{sh}}(\mathrm{GPa})$ & $U_{\mathrm{sh}}(\mathrm{m} / \mathrm{s})$ & $P_{\max }^{*}$ & $U_{\max }^{*}$ \\
\hline 1.1 & 0.19 & 106.9 & 8.36 & 9.58 \\
1.15 & 0.31 & 166.2 & 6.95 & 7.64 \\
1.2 & 0.43 & 220.5 & 6.23 & 6.62 \\
1.3 & 0.68 & 321.4 & 5.43 & 5.60 \\
1.4 & 0.90 & 399.7 & 4.80 & 5.13 \\
1.5 & 1.20 & 494.8 & 4.53 & 4.73 \\
1.6 & 1.53 & 587.9 & 4.35 & 4.39 \\
1.7 & 1.90 & 681.6 & 4.11 & 4.14 \\
1.8 & 2.20 & 751.0 & 3.95 & 4.03 \\
1.9 & 2.55 & 826.2 & 3.80 & 3.99 \\
\hline
\end{tabular}

Table 1 Maximum pressure and velocity reached during the bubble collapse for different incident shock intensity.

Evolutions of the maximum pressure $P_{\max }^{*}$ (left) and the maximum axial velocity $U_{\max }^{*}$ (right) with the Mach number of the incident shock $M_{\mathrm{sh}}$ are plotted 
in Figure 12. Using the numerical data, we propose two empirical laws which provide these maximum values reached during the bubble collapse. These relations are based on the observation that the product of maximum values with the quantity $\sqrt{M_{\mathrm{sh}}^{2}-1}$ vary quasi linearly with the Mach number shock. A linear approximation of these products give the following laws:

$$
P_{\max }^{*}=\frac{3.048 M_{\mathrm{sh}}+0.45}{\sqrt{M_{\mathrm{sh}}^{2}-1}} \quad \text { and } \quad U_{\max }^{*}=\frac{2.722 M_{\mathrm{sh}}+1.166}{\sqrt{M_{\mathrm{sh}}^{2}-1}}
$$

We can see in Figure 12 that these relations fit perfectly the numerical results.

\section{Concluding remarks}

This paper introduces a simple four-equation model for the simulation of a shock-bubble interaction leading to the cavity collapse. It is observed that most phenomenon associated to this interaction such as the liquid jet formation, the bubble division and the generation of a blast wave are significantly captured. Two pressure peaks are highlighted during the process: the first one when the blast wave is generated and the second one when the leftward wave front impacts the bubble fragments. This second peak is the most intense reached during the collapse.

The influence of the incident shock strength is studied. A case with a low Mach number shock $M_{\mathrm{sh}}=1.1$ is computed. We observe that the dimensionless maximum pressure increases as the incident shock wave becomes weaker. The topology of the flow is detailed. It is shown that a supplementary pressure peak appears in comparison with the high Mach number case due to the existence of a low velocity area (even a recirculation area) just behind the leftward wave front. This pressure peak constitutes the most intense reached during the bubble collapse.

Finally the evolution of the dimensionless maximum pressure with the Mach number of the incident shock wave is studied. An empirical law is proposed function of the quantity $\sqrt{M_{\mathrm{sh}}^{2}-1}$. It is shown that this relation perfectly suits the numerical results. A similar law is also proposed for the dimensionless maximum axial velocity.

The current work needs additional improvements. In future work, we plan to introduce mass transfer between phases and to perform three-dimensional simulations.

\section{Acknowledgments}

Authors would like to thank the reviewers for the constructive suggestions leading to substantial improvement of the text. 


\section{References}

1. Abgrall, R.: How to prevent pressure oscillations in multicomponent flow calculations: a quasi conservative approach. Journal of Computational Physics 125(1), 150-160 (1996). DOI 10.1006/jcph.1996.0085

2. Allaire, G., Clerc, S., Kokh, S.: A five-equation model for the simulation of interfaces between compressible fluids. Journal of Computational Physics 181(2), 577-616 (2002). DOI 10.1006/jcph.2002.7143

3. Apazidis, N.: Numerical investigation of shock induced bubble collapse in water. Physics of Fluids 28, 046,101 (2016). DOI 10.1063/1.4944903

4. Baer, M., Nunziato, J.: A two-phase mixture theory for the deflagration-to-detonation transition (DDT) in reactive granular materials. Int. Journal of Multiphase Flow 12, 861-889 (1986). DOI 10.1016/0301-9322(86)90033-9

5. Ball, G., Howell, B., Leighton, T., Schofield, M.: Shock-induced collapse of a cylindrical air cavity in water: a Free-Lagrange simulation. Shock Waves 10, 265-276 (2000). DOI 10.1007/s001930000060

6. Batten, P., Clarke, N., Lambert, C., Causon, D.: On the choice of wave speeds for the HLLC Riemann solver. SIAM J. Sci. Comput. 18(6), 1553-1570 (1997). DOI $10.1137 /$ S1064827593260140

7. Bourne, N.: On the collapse of cavities. Shock Waves 11, 447-455 (2002). DOI $10.1007 / \mathrm{s} 001930200128$

8. Coralic, V., Colonius, T.: Shock-induced collapse of a bubble inside a deformable vessel. European Journal of Mechanics B/Fluids 40, 64-74 (2013). DOI 10.1016/j.euromechflu.2013.01.003

9. Daude, F., Galon, P., Gao, Z., Blaud, E.: Numerical experiments using a HLLCtype scheme with ALE formulation for compressible two-phase flows five-equation models with phase transition. Computer \& Fluids 94, 112-138 (2014). DOI 10.1016/j.compfluid.2014.02.008

10. Goncalves, E.: Numerical study of expansion tube problems: Toward the simulation of cavitation. Computers \& Fluids 72, 1-19 (2013). DOI 10.1016/j.compfluid.2012.11.019

11. Goncalves, E., Charriere, B.: Modelling for isothermal cavitation with a fourequation model. International Journal of Multiphase Flow 59, 54-72 (2014). DOI 10.1016/j.ijmultiphaseflow.2013.10.015

12. Goncalves, E., Patella, R.F.: Numerical simulation of cavitating flows with homogeneous models. Computers \& Fluids 38(9), 1682-1696 (2009). DOI 10.1016/j.compfluid.2009.03.001

13. Goncalves, E., Zeidan, D.: Simulation of compressible two-phase flows using a void ratio transport equation. Communications in Computational Physics (in press)

14. Haas, J., Sturtevant, B.: Interaction of weak shock waves with cylindrical and spherical gas inhomogeneities. Journal of Fluid Mechanics (181), 41-76 (1987). DOI $10.1017 /$ S0022112087002003

15. Hawker, N., Ventikos, Y.: Interaction of a strong shockwave with a gas bubble in a liquid mediam: a numerical study. Journal of Fluid Mechanics 701, 59-97 (2012). DOI $10.1017 / \mathrm{jfm} .2012 .132$

16. Hu, X., Khoo, B., Adams, N., Huang, F.: A conservative interface method for compressible flow. Journal of Computational Physics 219, 553-578 (2006). DOI 10.1016/j.jcp.2006.04.001

17. Ishii, C., Hibiki, T.: Thermo-fluid dynamics of two-phase flow. Springer (2006). DOI 10.1007/978-1-4419-7985-8

18. Jamaluddin, A., Ball, G., Turangan, C., Leighton, T.: The collapse of single bubbles and approximation of the far-field acoustic emissions for cavitation induced by shock wave lithotripsy. Journal of Fluid Mechanics 677, 305-341 (2011). DOI 10.1017/jfm.2011.85

19. Johnsen, E., Colonius, T.: Numerical simulations of non-spherical bubble collapse. Journal of Fluid Mechanics 629, 231-262 (2009). DOI 10.1017/S0022112009006351

20. Kapila, A., Menikoff, R., Bdzil, J., Son, S., Stewart, D.: Two-phase modeling of deflagration-to-detonation transition in granular materials: reduced equations. Physics of fluids 13(10), 3002-3024 (2001). DOI 10.1063/1.1398042 
21. Kunz, R., Boger, D., Stinebring, D., Chyczewski, T., Lindau, J., Gibeling, H., Venkateswaran, S., Govindan, T.: A preconditioned Navier-Stokes method for two-phase flows with application to cavitation prediction. Computers \& Fluids 29(8), 849-875 (2000). DOI 10.1016/S0045-7930(99)00039-0

22. Lauer, E., Hu, X., Hickel, S., Adams, N.: Numerical investigation of collapsing cavity arrays. Physics of Fluids 24, 052,104 (2012). DOI 10.1063/1.4719142

23. Liu, T., Khoo, B., Yeo, K.: Ghost fluid method for strong shock impacting on material interface. Journal of Computational Physics 190(2), 651-681 (2003). DOI 10.1016/S00219991(03)00301-2

24. Massoni, J., Saurel, R., Nkonga, B., Abgrall, R.: Some models and Eulerian methods for interface problems between compressible fluids with heat transfer. Int. Journal of Heat ans Mass Transfer 45, 1287-1307 (2002). DOI 10.1016/S0017-9310(01)00238-1

25. Metayer, O.L., Massoni, J., Saurel, R.: Elaborating equations of state of a liquid and its vapor for two-phase flow models. Int. Journal of Thermal Sciences 43, 265-276 (2004). DOI 10.1016/j.ijthermalsci.2003.09.002

26. Murrone, A., Guillard, H.: A five equation reduced model for compressible two phase flows problems. Journal of Computational Physics 202(2), 664-698 (2005). DOI 10.1016/j.jcp.2004.07.019

27. Nourgaliev, R., Dinh, T., Theofanous, T.: Adaptive characteristics-based matching for compressible multifluid dynamics. Journal of Computational Physics 213, 500-529 (2006). DOI 10.1016/j.jcp.2005.08.028

28. Ozlem, M., Schwendeman, D., Kapila, A., Henshaw, W.: A numerical study of shockinduced cavity collapse. Shock Waves 22, 89-117 (2012). DOI 10.1007/s00193-0110352-9

29. Plesset, M., Chapman, R.: Collapse of an initially spherical vapour cavity in the neighbourhood of a solid boundary. Journal of Fluids Mechanics 47, 283-290 (1971). DOI $10.1017 /$ S0022112071001058

30. Shyue, K.: A volume-fraction based algorithm for hybrid barotropic and non-barotropic two-fluid flow problems. shock Waves 15, 407-423 (2006). DOI 10.1007/s00193-0060037-y

31. Terashima, H., Tryggvason, G.: A front-tracking/ghost-fluid method for fluid interfaces in compressible flows. Journal of Computational Physics 228(11), 4012-4037 (2009). DOI 10.1016/j.jcp.2009.02.023

32. Toro, E., Spruce, M., Speares, W.: Restoration of the contact surface in the HLLRiemann solver. Shock Waves 4, 25-34 (1994). DOI 10.1007/BF01414629

33. Zeidan, D.: Assessment of mixture two-phase flow equations for volcanic flows using Godunov-type methods. Applied Mathematics and Computation 272, 707-719 (2016). DOI 10.1016/j.amc.2015.09.038

34. Zheng, J., Khoo, B., Hu, Z.: Simulation of wave-flow-cavitation interaction using a compressible homogenous flow method. Commun. Comput. Phys 14(2), 328-354 (2013). DOI 10.4208/cicp.050412.140812a 


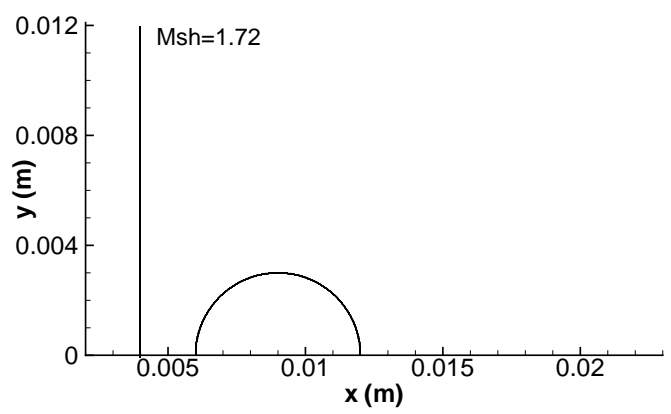

Fig. 1 Initial situation for the shock-bubble interaction $D_{0}=0.006 \mathrm{~m}$ and $M_{\mathrm{sh}}=1.72$. 

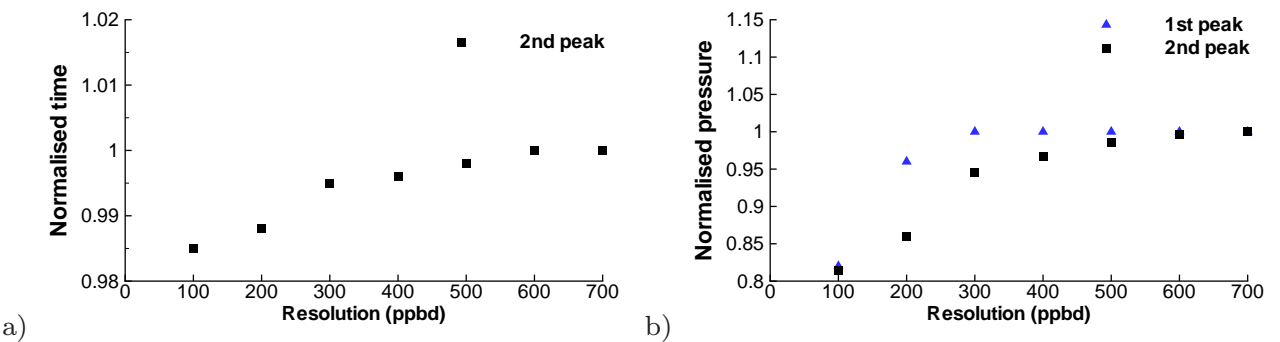

Fig. 2 Effect of the grid resolution: a) Second pressure peak time b) Pressure peaks during the collapse. Values are normalized by the value obtained with 700 nodes per bubble diameter. 


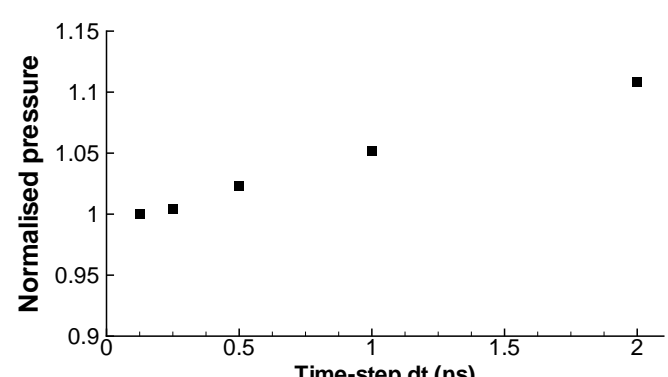

Fig. 3 Effect of the time step on the maximum pressure reached during the collapse. Values are normalized by the value obtained with $\Delta t=0.125 \cdot 10^{-9} \mathrm{~s}$. 


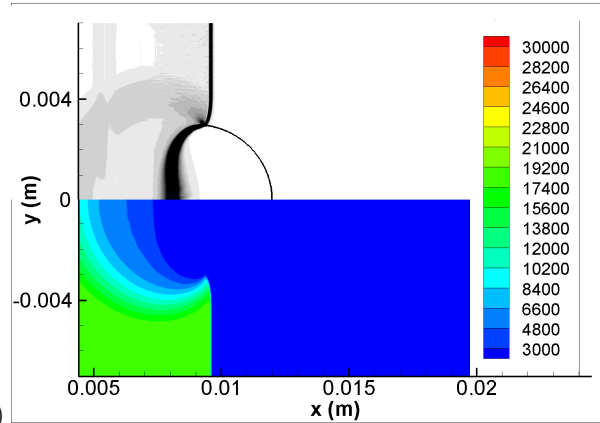

b)
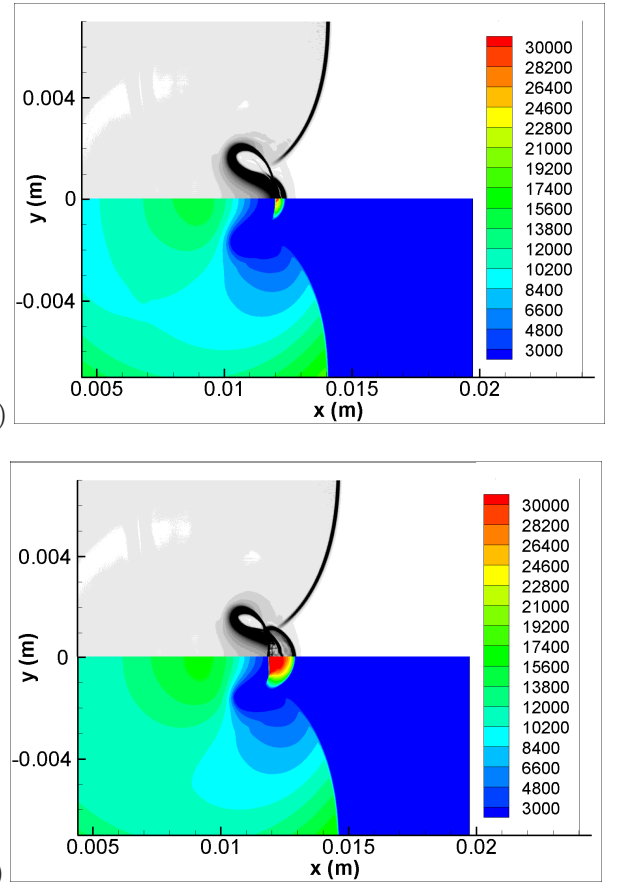

d)
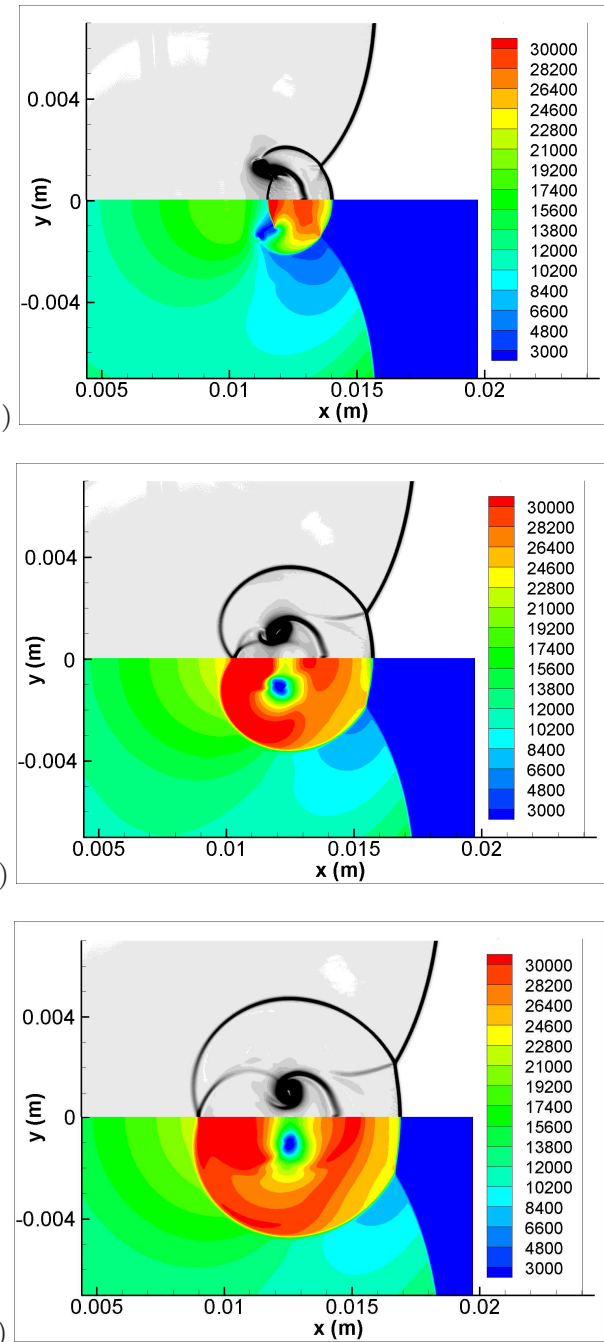

Fig. 4 Evolution of the density gradient modulus and pressure field (in bar) at times: a) $t=2 \mu \mathrm{s}, \mathrm{b}) t=3.6 \mu \mathrm{s}, \mathrm{c}) t=3.8 \mu \mathrm{s}, \mathrm{d}) t=4.2 \mu \mathrm{s}, \mathrm{e}) t=4.8 \mu \mathrm{s}$, and f) $t=5.2 \mu \mathrm{s}$. $D_{0}=0.006 \mathrm{~m}$ and $M_{\mathrm{sh}}=1.72$. 

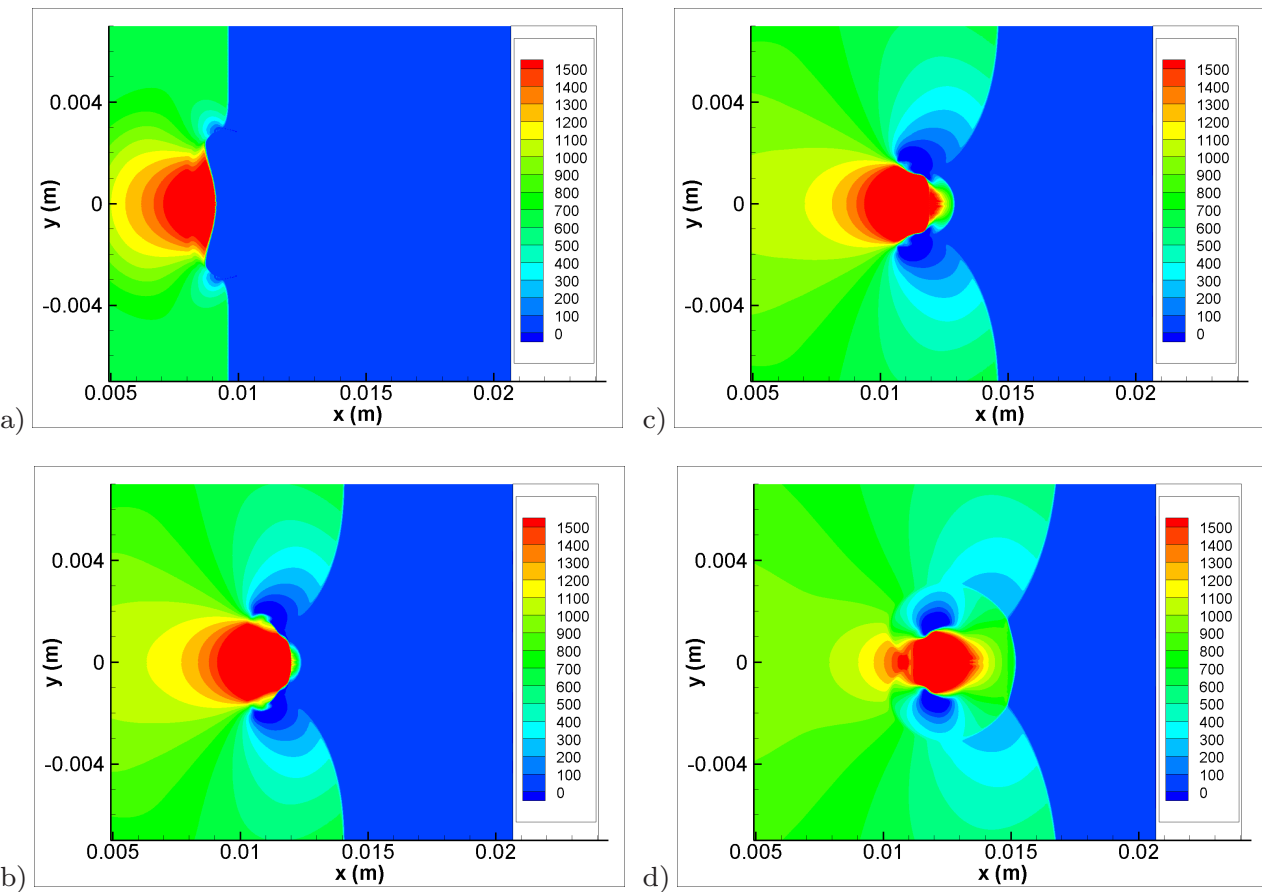

Fig. 5 Evolution of the axial velocity (in $\mathrm{m} / \mathrm{s}$ ) at times: a) $t=2 \mu \mathrm{s}, \mathrm{b}) t=3.6 \mu \mathrm{s}, \mathrm{c}$ ) $t=3.8 \mu \mathrm{s}, \mathrm{d}) t=4.6 \mu \mathrm{s} . D_{0}=0.006 \mathrm{~m}$ and $M_{\mathrm{sh}}=1.72$. 

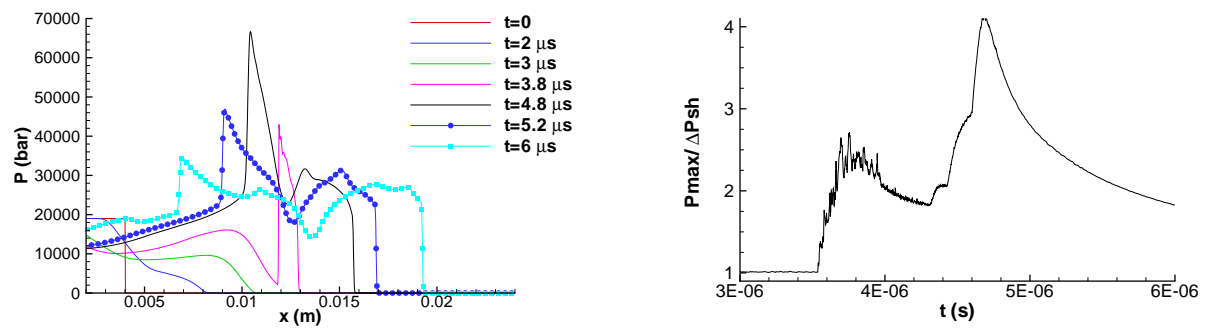

Fig. 6 Evolution of the pressure during the bubble collapse on the symmetry axis (left) and evolution of the dimensionless maximum pressure $P_{\max } / \Delta P_{\mathrm{sh}}$ during the collapse (right). $D_{0}=0.006 \mathrm{~m}$ and $M_{\mathrm{sh}}=1.72$. 

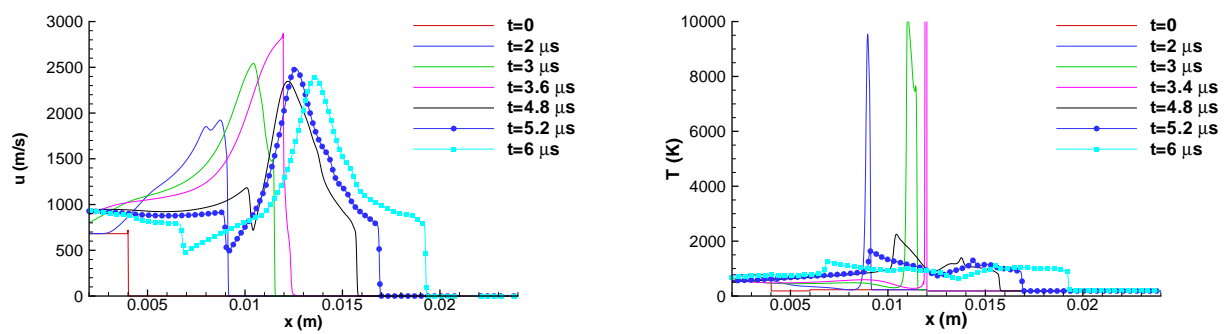

Fig. 7 Evolution of the axial velocity (left) and temperature on the symmetry axis (right). $D_{0}=0.006 \mathrm{~m}$ and $M_{\mathrm{sh}}=1.72$. 
a)
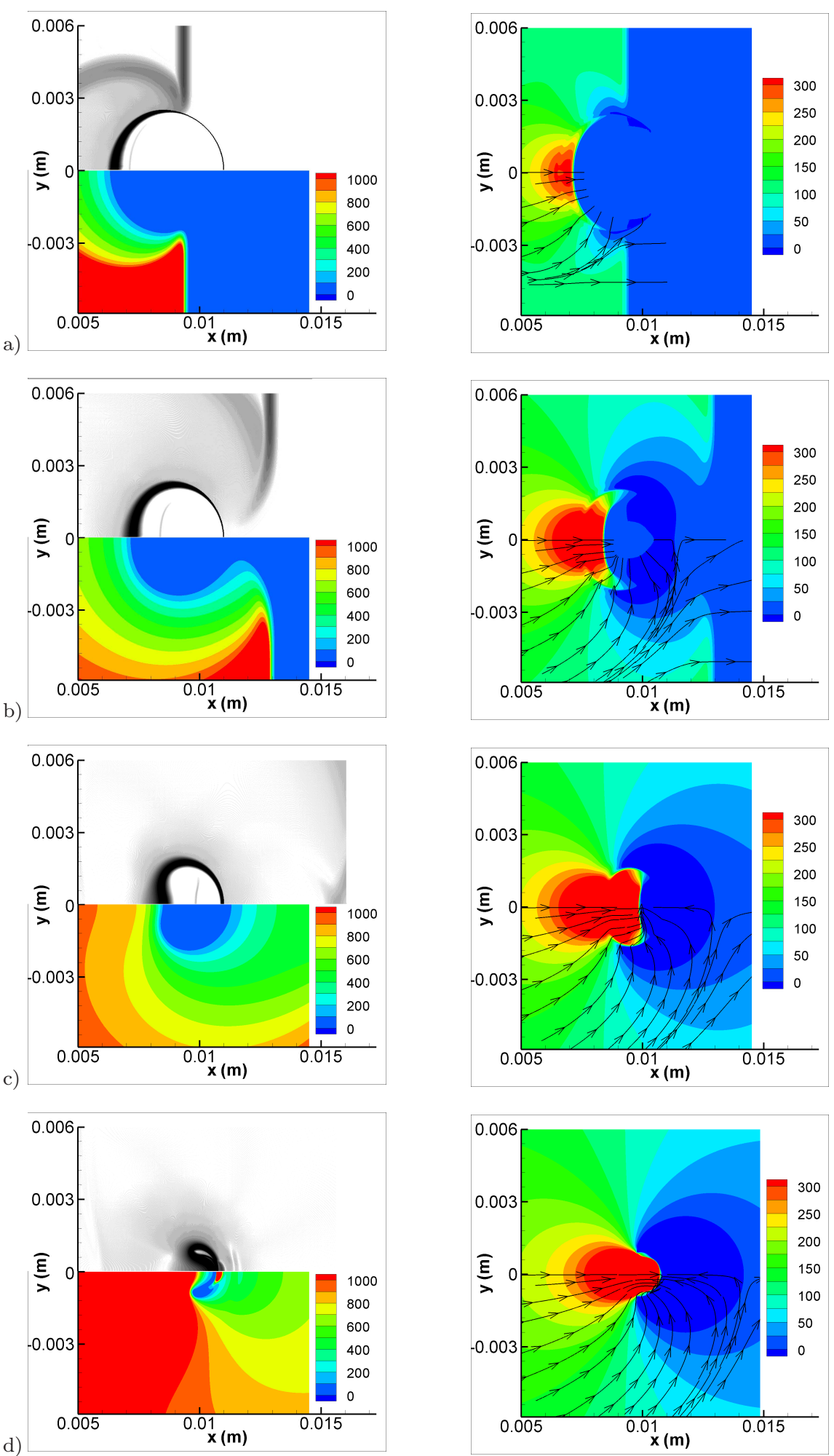

Fig. 8 Evolution of the density gradient modulus and pressure field (left) and axial velocity with streamlines at times: a) $t=3 \mu \mathrm{s}$, b) $t=5 \mu \mathrm{s}$, c) $t=7 \mu \mathrm{s}$ and d) $t=9.4 \mu \mathrm{s}$. $D_{0}=0.005$ $\mathrm{m}$ and $M_{\mathrm{sh}}=1.1$. 
e)
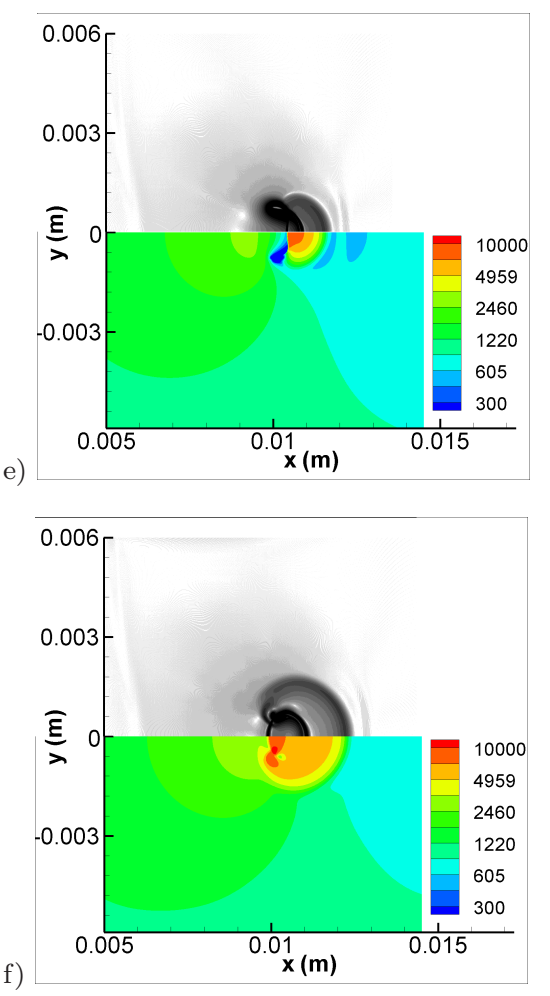

$$
\text { f) }
$$

g)
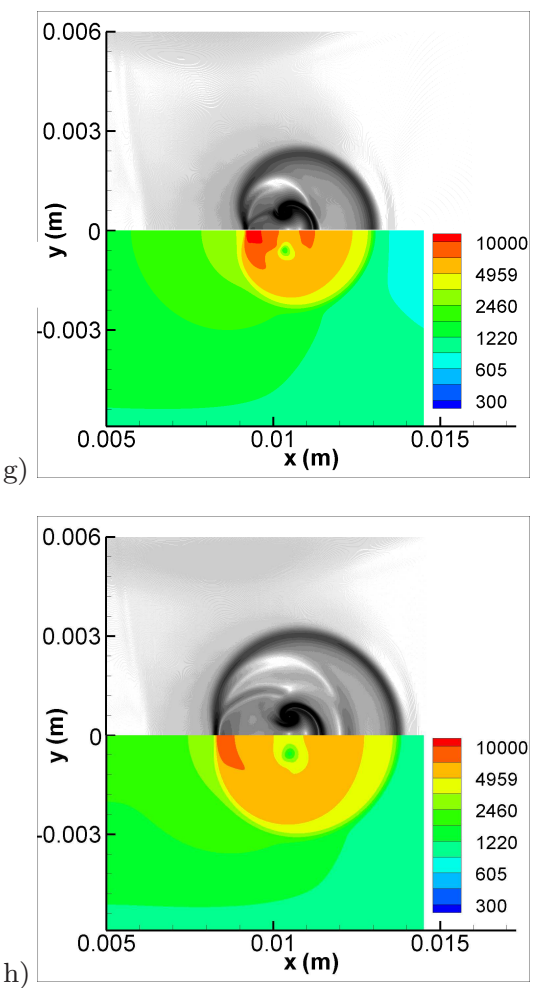
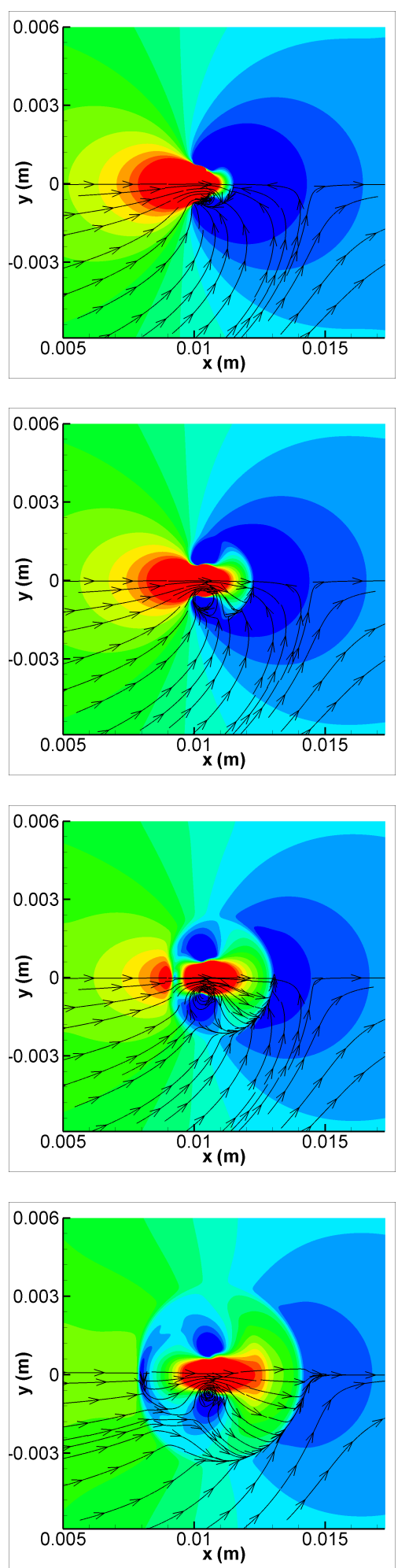

Fig. 9 Evolution of the density gradient modulus and pressure field (left) and axial velocity with streamlines at times: e) $t=9.8 \mu \mathrm{s}, \mathrm{f}) t=10.4 \mu \mathrm{s}, \mathrm{g}) t=10.8 \mu \mathrm{s}$, and h) $t=11.4 \mu \mathrm{s}$. $D_{0}=0.005 \mathrm{~m}$ and $M_{\mathrm{sh}}=1.1$. 

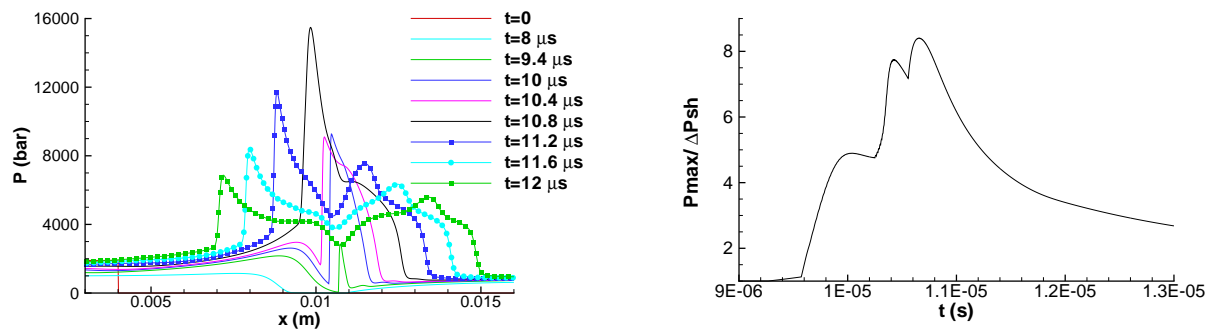

Fig. 10 Evolution of the pressure on the symmetry axis (left) and evolution of the maximum pressure $P_{\max }^{*}$ during the collapse (right). $D_{0}=0.005 \mathrm{~m}$ and $M_{\mathrm{sh}}=1.1$. 

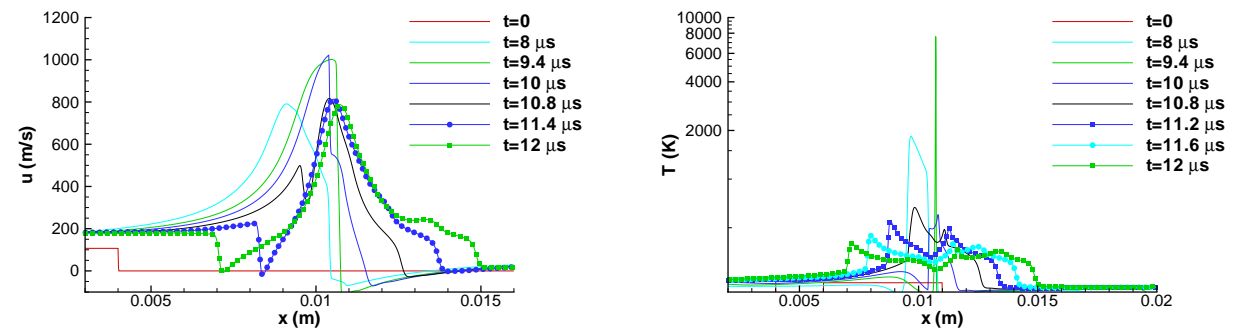

Fig. 11 Evolution of the axial velocity and the temperature on the symmetry axis (log scale) during the bubble collapse. $D_{0}=0.005 \mathrm{~m}$ and $M_{\mathrm{sh}}=1.1$. 

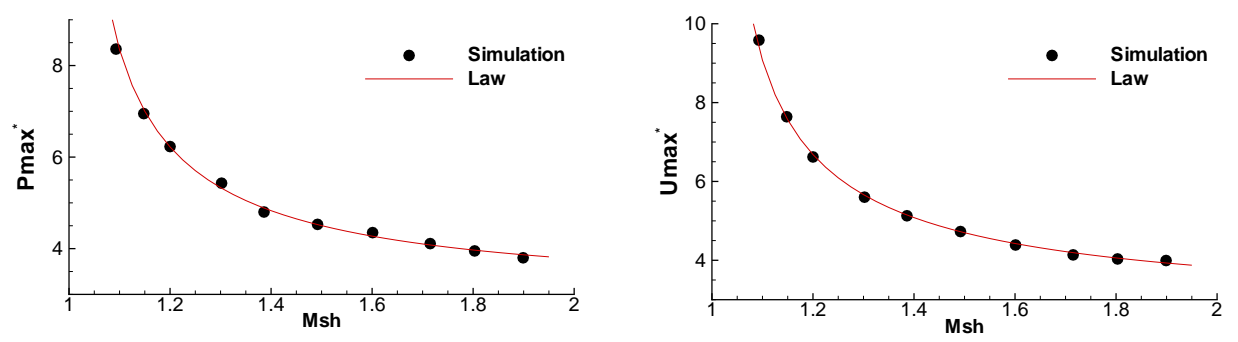

Fig. 12 Evolution of the maximum pressure $P_{\max }^{*}$ (left) and the maximum axial velocity $U_{\max }^{*}$ (right) with the incident shock Mach number $M_{\mathrm{sh}} \cdot D_{0}=0.006 \mathrm{~m}$. 